\title{
Oral Health in Children with Neurological Disorders
}

\author{
Priyanka Madaan ${ }^{1}$, Jitendra K Sahu ${ }^{2}$
}

\begin{abstract}
Pediatric neurological disorders encompass a wide range of disorders, including neurodevelopmental disorders, epilepsy, immunological disorders, neurometabolic disorders, degenerative disorders, and neuromuscular disorders. Oral health forms an integral part of holistic care in children with neurological disorders. Children with neurological disorders are predisposed to oral health problems because of a multitude of associated impairments, namely cognitive, communication, oro-motor, and behavioral. Children with neurodevelopmental, neurometabolic, neurodegenerative, and movement disorders have an increased risk of dental caries and worse gingival status due to the associated impairments. Children with epilepsy are prone to dental trauma and gingival overgrowth due to antiseizure medications. Children with neuromuscular disorders have limited physical mobility and often have facial or bulbar weakness resulting in drooling, swallowing difficulties, and malocclusion. The management of children with hereditary sensory autonomic neuropathy (associated with self-mutilation and dental concerns) is often challenging. Proper precautions need to be taken along with the involvement of an anesthesiologist during procedural sedation/ analgesia for children with neuromuscular disorders.

Keywords: Children, Dental, Neurological disorders, Oral health.

Journal of Postgraduate Medicine, Education and Research (2022): 10.5005/jp-journals-10028-1554
\end{abstract}

\section{INTRODUCTION}

Pediatric neurological disorders encompass a wide range of disorders, including neurodevelopmental disorders (NDDs), epilepsy, immunological disorders, neurometabolic disorders, degenerative disorders, and neuromuscular disorders. These constitute a major burden on pediatric healthcare requiring hospital visits, indoor as well as outdoor. These children are predisposed to oral health problems because of a multitude of associated impairments, namely cognitive-behavioral, communication, oro-motor, and motor disability. Dependence on caregivers for maintenance of oral hygiene, the possibility of neglect, immobility, hyperactivity, food selectivity, gastroesophageal reflux, and adverse effects of ongoing medication further add to this risk. Oral health forms an integral part of holistic care in children with these disorders. ${ }^{1}$ In this review, we will highlight important peculiarities and challenges associated with pediatric neurological disorders emphasizing a close liaison between pediatric neurologists and pediatric dentists to provide the best care. Table 1 provides an outline of common neurological disorders in children.

\section{Neurodevelopmental Disorders}

Diagnostic and Statistical Manual of Mental Disorders defines NDDs as a group of disorders with deficits leading to impaired functioning, with an onset in the developmental period. ${ }^{2,3}$ NDDs encompass several disorders, including intellectual disability (ID), autism spectrum disorder (ASD) and other communication disorders, attention-deficit hyperactivity disorder (ADHD), neurodevelopmental motor disorders, cerebral palsy (CP) and specific learning disorders. ${ }^{3,4}$ They are a significant public health burden in India. ${ }^{5}$ Children with NDDs suffer from different types of limitations ranging from cognitive impairment, social interaction, and communication problems, oro-motor impairments (reduced food clearance), attention and other behavioral and sensory issues. These limitations affect their ability to carry out activities of daily living, including self-care, eating, and maintenance of oral hygiene. Many children are entirely dependent on their caregivers for these
1,2Department of Pediatrics, Postgraduate Institute of Medical Education and Research, Chandigarh, India

Corresponding Author: Jitendra K Sahu, Department of Pediatrics, Postgraduate Institute of Medical Education and Research, Chandigarh, India, Phone: +91 8872066202, e-mail: jsh2003@gmail.com

How to cite this article: Madaan P, Sahu JK. Oral Health in Children with Neurological Disorders. J Postgrad Med Edu Res 2022;56(1):53-56. Source of support: Nil

Conflict of interest: None

activities. Hence, oral health problems are relatively more common in these children when compared with the typically developing children. ${ }^{1,6}$ Children with NDDs are also predisposed to nutritional deficiencies like vitamin $C$ and vitamin D deficiency etc., which further impact their oral health. These deficiencies may occur due to several reasons such as food selectivity and selective eating (because of sensory issues), feeding and swallowing difficulties, dietary restrictions, immobility, dependency, and parental neglect. ${ }^{7,8}$ Also, children with NDDs frequently require long-term medications or supplements, which are often administered as syrups considering the swallowing issues and difficulties in taking tablets. The continuous administration of sweetened liquid oral medicaments (syrups) adds to the risk of dental caries. ${ }^{9-11}$ Furthermore, some of the NDDs, their comorbidities, and drugs used in the management of comorbidities directly affect the teeth, gums, and oral health (e.g., kernicterus or chronic bilirubin encephalopathy is associated with enamel hypoplasia, comorbid gastroesophageal reflux disease predisposes teeth to gastric acid, prolonged phenytoin use in comorbid epilepsy is associated with gingival hyperplasia, anticholinergic medications used for movement disorders are associated with xerostomia). ${ }^{1,12,13}$ Access to oral healthcare services is another critical factor affecting oral health in children with NDDs. ${ }^{14}$ Recent systematic reviews and meta-analyses have revealed a higher burden of dental plaque, poor gingival status, and increased risk of dental caries in children

(c) The Author(s). 2022 Open Access This article is distributed under the terms of the Creative Commons Attribution 4.0 International License (https://creativecommons. org/licenses/by-nc/4.0/), which permits unrestricted use, distribution, and non-commercial reproduction in any medium, provided you give appropriate credit to the original author(s) and the source, provide a link to the Creative Commons license, and indicate if changes were made. The Creative Commons Public Domain Dedication waiver (http://creativecommons.org/publicdomain/zero/1.0/) applies to the data made available in this article, unless otherwise stated. 
Table 1: A brief overview of important pediatric neurological disorders in the context of dental health

- Neurodevelopmental disorders: A group of disorders with deficits leading to impaired functioning, with an onset in the developmental period.

- Intellectual disability (ID): Deficits in intellectual functioning and adaptive behavior; onset before the age of 18 years.

- Autism spectrum disorder (ASD): Characterized by restricted, repetitive behaviors and deficits in social-emotional reciprocity and nonverbal communication; onset in early childhood.

- Attention-deficit hyperactivity disorder (ADHD): A persistent pattern of inattention and/or hyperactivity-impulsivity; features present before the age of 12 years.

- Neurodevelopmental motor disorders: With predominant deficits in developmental and motor functioning such as Tic disorder.

- Epilepsy: Characterized by unprovoked recurrent seizures (episodes of sensory or motor disturbance, impaired consciousness occurring due to abnormal excessive electrical activity in the brain).

- Neurometabolic disorders: Conditions causing neurological problems secondary to inborn errors of metabolism such as enzyme deficiency, for example, Lesch - Nyhan syndrome.

- Neurodegenerative disorders: Progressive neurological disorders occurring as a result of progressive loss of structure or function of neurons leading to cell death.

- Disorders of movement and balance: The prototype is cerebral palsy which is a disorder of movement, tone, posture, and balance occurring as a result of insult to the developing brain.

- Neuromuscular disorders: For the sake of simplicity, these include muscle disorders (myopathies, muscular dystrophies), neuromuscle junction disorders (myasthenia), anterior horn cell disorders (spinal muscular atrophy), and nerve disorders (hereditary and acquired neuropathies). Hereditary sensory autonomic neuropathy (HSAN) is associated with self-mutilation and dental concerns (due to lack of pain sensation).

and adolescents with intellectual disability, CP, ADHD, and ASD. ${ }^{15-19}$ Children with CP also have an increased risk of dental malocclusion and anterior open bite, while children with ADHD are predisposed to traumatic dental injuries. ${ }^{16,17}$

Besides the behavioral concerns (such as fear of separation and procedural stimuli like loud noises, darkness, injections, etc.) seen in typically developing children during dental care visits, children with NDDs may have several other issues. ${ }^{20}$ Behavior interventions such as graduated exposure to fearful stimuli, distraction/relaxation, positive reinforcement, desensitization strategies (especially for children with ASD with sensory issues), etc., might help tackle these behavioral concerns and improve compliance. ${ }^{20,21}$ For highly uncooperative children and adolescents with NDDs with severe behavioral issues, dental interventions/procedures under sedation or general anesthesia are effective management options. ${ }^{1,22}$

\section{Epilepsy}

Childhood epilepsy is a global health problem, and its prevalence is nearly $6.2 / 1,000$ population. ${ }^{23}$ There are various epilepsy syndromes that present at different ages, and their classification is based on age at presentation, seizure type, and electroencephalographic findings. Various seizure types include generalized onset (tonic-clonic, clonic-tonic, myoclonic, absence, atonic, spasms etc.), focal onset (automatisms, autonomic, arrest, atonic, clonic, hyperkinetic etc.) and unknown onset. Children with epilepsy are at risk of dental trauma due to generalized seizure, associated teeth grinding and fall associated injuries, especially common in children with drop attacks due to myoclonic, atonic and tonic seizures. They routinely receive antiseizure medications such as phenytoin, valproate, phenobarbitone, levetiracetam etc. Long-term use of phenytoin is commonly associated with gingival overgrowth, which is partially preventable by the adjunct use of oral folic acid. ${ }^{24}$ Phenytoin and carbamazepine are also associated with the alveolar bone loss. ${ }^{25}$ While the dental procedure is planned, it is important that children with epilepsy should not have missed the regular dose of antiseizure medications. There is also a theoretical risk of seizure occurrence during the dental procedure, and therefore, dentists should be aware of the management of acute seizures.

\section{Neurometabolic and Neurodegenerative Disorders (Table 2)}

There are various inborn errors of metabolism and neurodegenerative disorders, which either present as episodic or a chronic progressive regression of acquired abilities. Individually, these are rare, but these disorders form a significant bulk when combined, and their management is very challenging. These disorders are very heterogeneous and usually have an underlying genetic explanation.

Broadly, neurometabolic disorders can be divided into small molecule disorders (such as urea cycle disorder, amino acidopathies, etc.), large molecule disorders (including storage disorders e.g., lysosomal storage disorder, peroxisomal disorders), disorders of cerebral energy metabolism, and miscellaneous metabolic disorders (Table 2). Based on the affected metabolic pathway, neurometabolic disorders can be classified as urea cycle disorders, fatty acid oxidation defects, organic acidemias, aminoacidopathies, peroxisomal disorders, lysosomal disorders, glycosylation disorders, disorders of cholesterol biosynthesis, mitochondrial disorders, and neurotransmitter disorders. Most of the children with these disorders either present as acute encephalopathy or chronic progressive neurological disorders with or without organomegaly (seen in many storage disorders). The major oral health concerns in children with these disorders include poor oral hygiene, pseudo-bulbar palsy, spastic jaw, oromotor dystonia, etc. These children often require special dietary management requiring frequent feeding, carbohydrate-rich diet etc., to optimize normal growth and development, which can predispose them to dental caries. ${ }^{26}$ Lesch - Nyhan syndrome, a disorder of purine metabolism, needs special mention as children may often present with self-mutilation.

Neurodegenerative disorders are progressive neurological disorders occurring due to progressive loss of structure or function of neurons leading to cell death. They can be classified as gray matter degenerative disorders and white matter degenerative disorders. Gray matter disorders primarily affect neuronal cell bodies and present with epilepsy and progressive cognitive decline, for example, neuronal ceroid lipofuscinosis, while white matter disorders affect myelinated structures and present with progressive spasticity. White matter disorders can be further classified into hypomyelination disorders [such as Pelizaeus Merzbacher disease, $4 \mathrm{H}$ syndrome (hypomyelination, hypogonadotropic, hypogonadism and hypodontia syndrome)] and demyelinating disorders such as metachromatic leukodystrophy. Except for hypodontia, the affected children have oral health concerns very similar to neurodevelopmental and movement disorders depending on cognitive and motor limitations. 
Table 2: Broad classification of neurometabolic and neurodegenerative disorders

\section{- Neurometabolic disorders}

- Small molecule disorders: Interfere intermediary metabolism of amino acids, organic acids, fatty acids and ammonia.

- Urea cycle disorders ( $\mathrm{N}$-acetylglutamate synthase deficiency, ornithine transcarbamylase deficiency, citrullinemia, arginase deficiency, etc.).

- Fatty acid oxidation disorders (medium-chain acyl CoA dehydrogenase deficiency, very long-chain acyl CoA dehydrogenase deficiency, short-chain acyl CoA dehydrogenase deficiency, etc.).

- Organic acidemias (methylmalonic acidemia, propionic acidemia, etc.).

- Aminoacidopathies (Phenylketonuria, maple syrup urine disease etc.).

- Large molecule disorders: Interfere metabolism of complex molecules.

- Lysosomal storage disorders (Gaucher disease, Batten disease, GM1- gangliosidosis, GM2- gangliosidosis, etc.).

- Peroxisomal disorders (Zellweger syndrome, Refsum's disease, etc).

- Disorders of glycosylation.

- Disorders of complex lipid metabolism (hereditary sensory autonomic neuropathy, etc).

- Cerebral energy, metabolism disorders: Mitochondrial energy, metabolism defects, glucose transporter defects.

- Miscellaneous: Carbohydrates, purines and pyrimidines, and neurotransmitters related disorders, etc.

- Neurodegenerative disorders

- Gray matter degenerative brain disorder: When the degenerative process primarily involved neuronal cell bodies (cortex, basal ganglia primarily affected). Present with epilepsy and progressive cognitive decline, for example, neuronal ceroid lipofuscinosis.

- White matter degenerative brain disorder: When the degenerative process primarily involves myelinated structures/axons (white matter primarily affected). Present with progressive spasticity, vision loss, hearing impairment, for example, leukodystrophies.

- Other disorders such as ataxia telangiectasia (a progressive cerebellar disorder associated with immunodeficiency and chromosomal breakage) - Dental radiographs should be avoided due to a risk of malignancy due to radiation exposure.

\section{Immune-mediated Neurological Disorders}

There are various disorders under the umbrella term of immune-mediated neurological disorders. However, chronic relapsing disorders such as autoimmune encephalitis, neuromyelitis optica, and multiple sclerosis are important from a dentist point of view. These children often have episodes of recurrent neurological impairment due to flaring up of underlying immunological dysfunction, and they usually receive immunosuppressant medications. It is crucial to use adjunct antibiotics after a dental procedure in children on immunosuppression because they are prone to severe infections.

\section{Movement Disorders}

Movement disorders including tics, tremors, ataxia, dystonia, and chorea are common in various acquired and genetic neurological disorders. Dystonia deserves special attention from dentists because of the associated oral health concerns. It is characterized by
Table 3: Oral health problems and their predisposing factors in children with neurological disorders

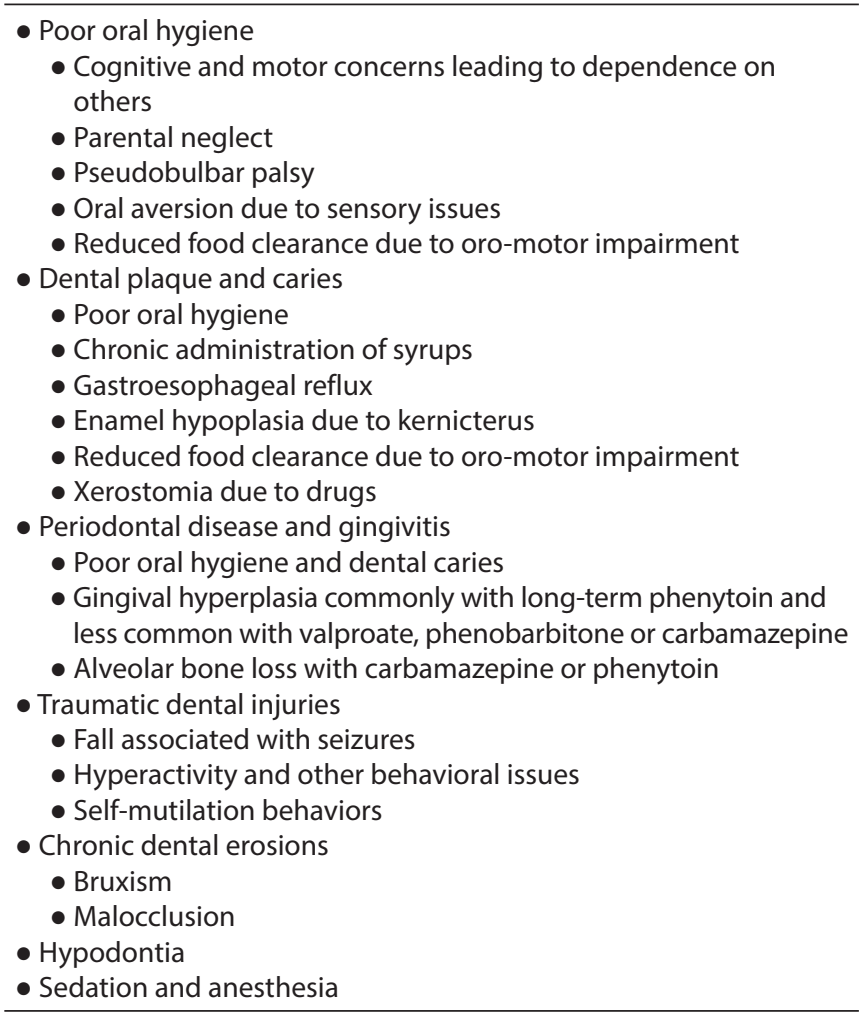

abnormal involuntary twisting postures and is commonly present in a dystonic variant of $\mathrm{CP}$ and various genetic neurological disorders, for example, pantothenate kinase-associated neurodegeneration, neuronal brain iron accumulation etc. Dentists should be aware that dystonia can worsen with stress while a child is nervous on a dental chair. So, it is essential to give extra effort to make such a child completely relaxed and comfortable. Also, the anticholinergic medications such as trihexyphenidyl used for dystonia can cause dry mouth, which along with oro-motor dystonia, can lead to reduced food clearance and poor oral health. ${ }^{1}$

\section{Neuromuscular Disorders}

These are heterogeneous disorders classified based on the site of involvement-muscular dystrophies and congenital myopathies with muscle localization, spinal muscular atrophies with anterior horn localization, myasthenia gravis with neuromuscular junction involvement, and genetic or acquired neuropathies with involvement of peripheral nerves. Children with these disorders often have limited physical mobility and facial or bulbar weakness resulting in drooling and swallowing difficulties. Dental malocclusions may occur in children with congenital myopathies with facial weakness. Children with hereditary sensory and autonomic neuropathy have pain insensitivity and can present with self-mutilation and pose a management challenge to the dentist team. Children with myasthenia gravis have difficulty in prolonged opening of the mouth, and therefore, the dental procedure should be brief and quick. It should be noted that oral anticholinesterase medications commonly used in myasthenia gravis increase salivation.

General anesthesia is often contraindicated in the presence of respiratory muscle weakness. The concern with general 
anesthesia is high in children with neuromuscular disorders and comorbid cardiomyopathy. There is also a susceptibility risk for malignant hyperthermia in some congenital myopathies and dystrophies. ${ }^{27}$ Local anesthesia is the preferred choice when needed. Procaine as anesthetic agent should be avoided in children with myasthenia gravis. Table 3 lists the critical oral health problems and their predisposing factors in children with neurological disorders.

\section{SUMMARY}

To summarize, oral health management of children with neurological disorders is challenging and need to be dealt with special care. The issue of oral health care is often missed in these children because of ongoing medical issues. As highlighted, these children are at higher risk of oral health problems, it is crucial that medical care providers should be aware of these aspects, and oral examination should not be missed during routine outdoor and indoor patient care services. A timely referral to oral health care providers is of paramount importance. Medical and dental health care providers of children with neurological disorders should work as a team, and thorough planning in advance for complex cases would be very helpful. Interprofessional collaborations between dentists, pediatric neurologists, psychologists, and occupational therapists might help provide care to children with neurological disorders.

\section{References}

1. Norwood KW, Slayton RL. Oral health care for children with developmental disabilities. Pediatrics 2013;131(3):614-619. DOI: $10.1542 /$ peds.2012-3650

2. American Psychiatric Association. Diagnostic and Statistical Manual of Mental Disorders. Arlington, VA: American Psychiatric Association Publishing; 2013.

3. Morris-Rosendahl DJ, Crocq MA. Neurodevelopmental disorders- the history and future of a diagnostic concept. Dialogues Clin Neurosci 2020;22(1):65-72. DOI: 10.31887/DCNS.2020.22.1/macrocq.

4. Thapar A, Cooper M, Rutter M. Neurodevelopmental disorders. Lancet Psychiatry 2017;4(4):339-346. DOI:10.1016/S2215-0366(16)30376-5.

5. Arora NK, Nair MKC, Gulati S, et al. Neurodevelopmental disorders in children aged 2-9 years: population-based burden estimates across five regions in India. PLoS Med 2018;15(7):e1002615. Published 2018. DOI:10.1371/journal.pmed.1002615

6. Lewis CW. Dental care and children with special health care needs: a population-based perspective. Acad Pediatr 2009;9(6):420-426. DOI: 10.1016/j.acap.2009.09.005

7. Madaan P, Krishnappa A, Yadav J, et al. Selective eating: a common fuss in neurodevelopmental disorders. Pediatr Neurol 2019;95:90. DOI: 10.1016/j.pediatrneurol.2019.01.007

8. Yadav J, Madaan P, Jain V. Brown tumor due to vitamin D deficiency in a child with cerebral palsy. Indian J Pediatr 2014;81(12):1419. DOI: 10.1007/s12098-014-1517-1

9. Pomarico L, Souza IP, Rangel Tura LF. Sweetened medicines and hospitalization: caries risk factors in children with and without special needs. Eur J Paediatr Dent 2005;6(4):197-201. PMID: 16426119.
10. Goyal A, Bhadravathi MC, Kumar A, et al. Comparison of dental caries experience in children suffering from epilepsy with and without administration of long term liquid oral medication. J Clin Diagn Res 2016;10(6):ZC78-ZC82. DOI: 10.7860/JCDR/2016/18211.8024

11. Al Humaid J. Sweetener content and cariogenic potential of pediatric oral medications: a literature. Int J Health Sci (Qassim) 2018;12(3):75-82. PMID: 29896075. PMCID: PMC5969777.

12. Madaan P, Reddy C, Saini L. Gaze Palsy: an important diagnostic clue J Pediatr 2019;212:236. DOI: 10.1016/j.jpeds.2019.03.048.

13. Asadi-Pooya AA, Rostaminejad M, Zeraatpisheh Z, et al. Cosmetic adverse effects of antiseizure medications; a systematic review. Seizure 2021;91:9-21. DOI: 10.1016/j.seizure.2021.05.010.

14. Ummer-Christian R, lacono T, Grills N, et al. Access to dental services for children with intellectual and developmental disabilities - a scoping review. Res Dev Disabil 2018;74:1-13. DOI: 10.1016/j. ridd.2017.12.022

15. Zhou Ni,Wong HM, Wen YF, et al. Oral health status of children and adolescents with intellectual disabilities: a systematic review and meta-analysis. Dev Med Child Neurol 2017;59(10):1019-1026. DOI: $10.1111 / \mathrm{dmcn} .13486$

16. Bensi C, Costacurta M, Docimo R. Oral health in children with cerebral palsy: a systematic review and meta-analysis. Spec Care Dentist 2020;40(5):401-411. DOI: 10.1111/scd.12506

17. Chau YCY, Peng SM, Mcgrath CPJ, et al. Oral health of children with attention deficit hyperactivity disorder: systematic review and meta-analysis. J Atten Disord 2020;24(7):947-962. DOI: $10.1177 / 1087054717743331$

18. Lam PP, Du R, Peng S, et al. Oral health status of children and adolescents with autism spectrum disorder: a systematic review of case-control studies and meta-analysis. Autism 2020;24(5):1047-1066. DOI: $10.1177 / 1362361319877337$

19. da Silva SN, Gimenez T, Souza RC, et al. Oral health status of children and young adults with autism spectrum disorders: systematic review and meta-analysis. Int J Paediatr Dent 2017;27(5):388-398. DOI: $10.1111 /$ ipd.12274

20. Kupzyk S, Allen KD. Pediatric prevention: oral health care. Pediatr Clin North Am 2020;67(3):513-524. DOI: 10.1016/j.pcl.2020.02.006.

21. Como DH, Stein Duker LI, Polido JC, et al. Oral health and autism spectrum disorders: a unique collaboration between dentistry and occupational therapy. Int J Environ Res Public Health 2020;18(1):135. DOI: 10.3390/ijerph18010135.

22. Wilson NJ, Lin Z, Villarosa A, et al. Countering the poor oral health of people with intellectual and developmental disability: a scoping literature review. BMC Public Health 2019;19(1):1530. DOI: 10.1186/ s12889-019-7863-1.

23. Pandey S, Singhi P, Bharti B. Prevalence and treatment gap in childhood epilepsy in a north Indian city: a community-based study J Trop Pediatr 2014;60(2):118-123. DOI: 10.1093/tropej/fmt091

24. Arya R, Gulati S, Kabra M, et al. Folic acid supplementation prevents phenytoin-induced gingival overgrowth in children. Neurology 2011;76(15):1338-1343. DOI: 10.1212/WNL.0b013e3182152844

25. Cornacchio AL, Burneo JG, Aragon CE. The effects of antiepileptic drugs on oral health. J Can Dent Assoc 2011;77:b140. PMID: 22260801.

26. Cleary MA, Francis DE, Kilpatrick NM. Oral health implications in children with inborn errors of intermediary metabolism: a review. Int J Paediatr Dent 1997;7(3):133-141. DOI: 10.1046/j.1365263x.1997.00229.x.

27. Litman RS, Griggs SM, Dowling JJ, et al. Malignant hyperthermia susceptibility and related diseases. Anesthesiology 2018;128(1): 159-167. DOI: 10.1097/ALN.0000000000001877. 